\title{
Surgical Management of Graves' Disease over a 25-year Period in a Single Institution: Comparison of Outcomes between Subtotal Thyroidectomy and Total Thyroidectomy
}

\author{
${ }^{1}$ Su A Lui, ${ }^{2}$ Jun L Teh, ${ }^{3}$ Yong X Gwee, ${ }^{4}$ Clifton Tay, ${ }^{5}$ Jesse Hu, ${ }^{6}$ Kee Y Ngiam, ${ }^{7}$ Charles TK Tan, ${ }^{8}$ Rajeev Parameswaran
}

${ }^{9}$ Abu Rauff, ${ }^{10}$ Wee B Tan, ${ }^{11}$ Wei K Cheah

\begin{abstract}
Introduction: Surgery is offered to patients with Graves' disease (GD) refractory to medical or radio-iodine therapy. Total thyroidectomy (TT) has been shown to lower recurrence rates of hyperthyroidism but subtotal thyroidectomy (ST) is thought to be associated with lower risk of postoperative complications. The aim of this study was to compare the postoperative outcomes of TT vs ST in the Singaporean population and identify risk factors that might predispose to treatment failure after ST.
\end{abstract}

Materials and methods: This is a retrospective review of consecutive patients who underwent surgery for GD at a single institution in Singapore. Patients who underwent thyroid surgery for GD between January 1991 and December 2015 were included in the study. The primary outcomes studied were rates of recurrent hyperthyroidism, hypocalcemia, recurrent laryngeal nerve injury, and length of stay postsurgery. The secondary outcome was to compare whether preoperative thyroid function and antibody levels predicted for treatment failure post-ST.

Results: Over a 25-year period, 79 patients underwent ST and 38 patients underwent TT. The rate of hyperthyroidism relapse post-ST was $20.3 \%$ compared with $0 \%$ post-TT ( $p<0.01$ ). There was no difference between the length of stay, or recurrent laryngeal nerve injury between patients who underwent ST compared with TT. Preoperative antibody levels and biochemical hyperthyroidism did not predict treatment failure after ST.

Conclusion: Total thyroidectomy is a more definitive surgical procedure for patients with GD refractory to medical therapy with lower risk of disease relapse and similar temporary and long-term risk of complications when compared with ST.

Keywords: Graves' disease, Outcome, Thyroidectomy.

How to cite this article: Lui SA, Teh JL, Gwee YX, Tay C, Hu J, Ngiam KY, Tan CTK, Parameswaran R, Rauff A, Tan WB, Cheah WK. Surgical Management of Graves' Disease over a 25-year Period in a Single Institution: Comparison of Outcomes

\footnotetext{
${ }^{1,3,4}$ Resident, ${ }^{2}$ Senior Resident, ${ }^{5,6,10}$ Consultant, ${ }^{7-9,11}$ Senior Consultant

1-4,6-10Department of Surgery, National University Health System Singapore

${ }^{5,11}$ Department of Surgery, $\mathrm{Ng}$ Teng Fong General Hospital Singapore
}

Corresponding Author: Su A Lui, Resident, Department of Surgery, National University Health System, Singapore, e-mail: su_ann_lui@nuhs.edu.sg between Subtotal Thyroidectomy and Total Thyroidectomy. World J Endoc Surg 2018;10(2):103-107.

\section{Source of support: Nil}

Conflict of interest: None

\section{INTRODUCTION}

Graves' disease is the commonest cause of hyperthyroidism. It is an autoimmune disorder characterized by an enlarged goiter, hyperthyroidism, and ophthalmopathy. ${ }^{1}$ Untreated GD in the long term predisposes one to blindness, cardiovascular comorbidities, and osteopenia and may carry significant morbidity and mortality. Treatment options for GD include antithyroid medications, radioiodine, or surgery. Indications for surgery in patients with GD include failure of medical management to antithyroid medications or radio-iodine therapy, younger patients with high risk of recurrence following medical management, large goiter with compressive symptoms, nodular thyroid where malignancy may coexist, severe Graves' ophthalmopathy, and patients' preference. $^{2}$

There has been much controversy about the surgical management of GD. Surgical options for GD include bilateral ST, unilateral hemithyroidectomy with contralateral $\mathrm{ST}^{3-5}$ and TT. ${ }^{6-8}$ Recent guidelines have proposed TT as the procedure of choice for the surgical management of GD, ${ }^{9}$ but other studies ${ }^{10,11}$ have argued that ST, especially when performed with a remnant thyroid of less than $3 \mathrm{gm}^{12}$ may be associated with a low risk of recurrent hyperthyroidism. The ST is associated with lesser risk of hypoparathyroidism ${ }^{13,14}$ and possibly recurrent laryngeal nerve palsy. A Cochrane review found TT to be more effective in preventing recurrent hyperthyroidism compared with ST with greater risk of permanent hypoparathyroidism and no effect on recurrent laryngeal nerve palsy or regression of Graves' ophthalmopathy. ${ }^{15}$

There is a paucity of literature reporting the reasons leading to relapse of hyperthyroidism after surgical therapy for GD. Kuma et $\mathrm{al}^{16}$ reported that circulating levels of thyroid hormones, thyroid-stimulating antibody, and thyroid-stimulating hormone (TSH)-binding immunoglobulin (TBII) did not predict for thyroid status post-ST. Sugino et $\mathrm{al}^{17}$ reported that larger thyroid 
remnant size, antimicrosomal hemagglutination antibody and TBII predicted the need for TSH suppression within a year after ST.

The purpose of the study was to report our experience of thyroidectomy for GD in a Southeast Asian population as such data have not been previously reported in the literature; Further to that, we also aimed to elucidate the factors that predict treatment failure after ST in our population.

\section{MATERIALS AND METHODS}

\section{Study Design}

We utilized a historical cohort of consecutive patients undergoing thyroidectomy for GD in a tertiary university hospital. Data and outcomes were prospectively recorded in a paper or electronic database and examined retrospectively for the purpose of this study. Patients who underwent thyroid surgery for GD before 2006 received ST; those who underwent surgery after the year 2009 received TT. Patients who were operated on in the intervening years received either procedure.

\section{Setting/Participants}

Consecutive patients undergoing either ST or TT for GD in a specialist endocrine surgery unit between January 1991 and December 2015 formed the study population. Patients younger than 18 years of age and those with missing follow-up data were excluded from the study. All patients who were scheduled for surgery presented either to the endocrine surgery service directly or were referrals from endocrinology or primary care clinics. Patients were counseled for surgery when they had large goiters with obstructive symptoms, hyperthyroid symptoms refractory to antithyroid, and/or radio-iodine therapy, Graves' ophthalmopathy, allergies to antithyroid medication, or when there was suspicion of a concomitant malignant thyroid nodule.

Patient characteristics recorded included patient gender, ethnicity, preoperative laboratory results with $\mathrm{TSH}$, free thyroxine, antithyroid peroxidase, antithyroglobulin, $\mathrm{TSH}$ receptor antibody, and preoperative thyroid volume as estimated by ultrasonography. The time of diagnosis until surgery, type of surgery performed, duration of surgery, and volume of remnant thyroid when ST was performed were also recorded.

\section{Surgical Technique}

All patients underwent surgery under general anesthesia without cervical block.

For TT, subplatysmal flaps were raised following a transverse skin crease incision two finger breaths above the sternal notch. The cervical linea alba was then divided to gain access to the thyroid gland; strap muscles are not routinely divided. The dissection was then carried out anterolaterally toward the carotid sheath where the middle thyroid vein was ligated between ties. Subsequently, the superior pole was identified and the superior pedicle was divided between either ties or an energy device. The parathyroid glands and recurrent laryngeal nerve are routinely identified in the procedure. Dissection was then carried out in the cricothyroid space, taking care to preserve the contralateral recurrent laryngeal nerve and parathyroid glands. For TT, all visible thyroid tissues were excised. All other patients underwent bilateral ST in which 2 to $4 \mathrm{gm}$ of normal thyroid tissue was left behind in the thyroid bed on each side. No patients underwent hemithyroidectomy or Dunhill procedure in this surgical series. Neuromonitoring was not used before the year 2011. All cases of thyroidectomy were performed with neuromonitoring since the year 2012 after change in the unit's thyroidectomy protocol.

\section{Study Outcomes}

Given that the current literature supports TT as the procedure of choice in GD patients undergoing surgical therapy, the primary outcomes examined for this study were the mean length of stay, incidence of hypocalcemia (temporary and permanent), hoarseness, and recurrent laryngeal nerve injury (temporary and permanent), and relapse of hyperthyroidism postsurgery. As there was no consensus in the current literature what constitutes early or late complications, we took arbitrarily complications persisting more than 6 months to be permanent and those that resolved within 6 months as temporary complications. Patients were considered hypocalcemic when corrected serum calcium was less than $2.1 \mathrm{mmol} / \mathrm{L}$ or when they experienced symptoms suggestive of hypocalcemia. Treatment failure or relapse was considered to have occurred if patients were persistently biochemically hyperthyroid or if they continued to exhibit clinical symptoms of hyperthyroidism after thyroidectomy. We do not routinely perform radio-iodine scans after surgery.

We also wanted to study whether any factor might predict treatment failure after ST. The secondary outcome was to examine if preoperative thyroid volumes, remnant gland volumes, preoperative thyroid function tests, and antibodies levels predicted treatment failure in the subgroup of patients who underwent ST.

\section{Statistics}

All categorical variables were compared using chisquare tests with a p-value of less than 0.05 considered 
statistically significant. The means of continuous variables, such as length of stay and laboratory results were compared using two-tailed Student's t test. Statistical analysis was performed using Statistical Package for the Social Sciences version 21 (IBM SPSS Statistics for Macintosh, version 21.0).

\section{RESULTS}

\section{Demographics}

A total of 130 patients underwent thyroid surgery in the 25-year period between January 1991 and December 2015. Of these, 13 patients were excluded due to missing follow-up data (Flow Chart 1). Patient demographics, as stratified according to whether they underwent ST or TT, is displayed in Table 1; 75 patients underwent bilateral ST before year 2006 and 27 patients who underwent surgery after the year 2009 received TT. In the intervening years, 11 out of 15 patients diagnosed with GD received TT.

Flow Chart 1: Patients undergoing surgery for Graves' disease

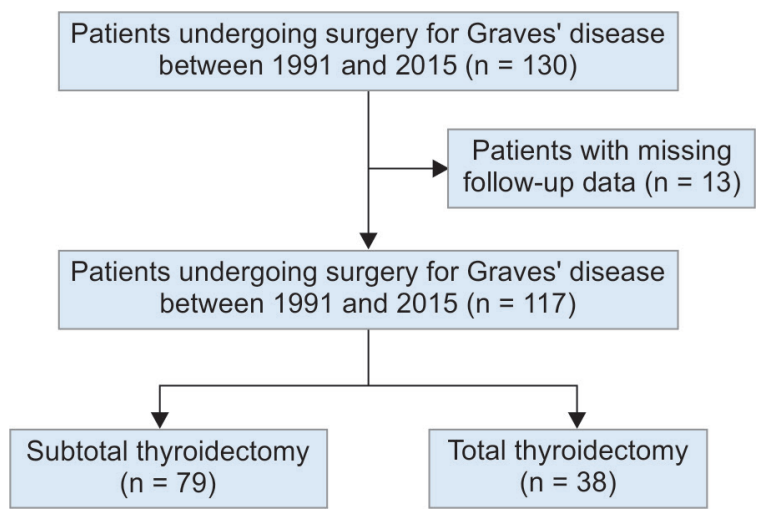

Table 1: Demographics of patients, stratified by ST vs TT

\begin{tabular}{llll}
\hline Demographics & $S T(\%)(n=79)$ & $T T(\%)(n=38)$ & $p$-value \\
\hline Gender & & & \\
Male & $10(12.6 \%)$ & $7(18.4 \%)$ & \\
Female & $69(87.3 \%)$ & $31(81.5 \%)$ & \\
Race & & & \\
Chinese & $73(92.4 \%)$ & $28(73.6 \%)$ & \\
Malay & $4(5.1 \%)$ & $10(26.3 \%)$ & \\
Indian & $1(1.3 \%)$ & 0 & 0.001 \\
Others & $1(1.3 \%)$ & 0 & 0.305 \\
Age & $31.3 \pm 7.3$ & $37.4 \pm 11.4$ & \\
Preoperative TSH & $0.73 \pm 2.830$ & $0.25 \pm 0.54$ & \\
levels (mIU/L) & & & \\
$\begin{array}{l}\text { Preoperative } \\
\text { thyroxine levels }\end{array}$ & $52.1 \pm 103$ & $16.9 \pm 4.5$ & 0.038 \\
(pmol/L) & & & 0.033 \\
Size of gland & $135 \pm 105$ & $94.4 \pm 60.2$ & \\
\hline
\end{tabular}

\section{Thyroid Status Postsurgery}

Three patients had persistent hyperthyroidism post-ST. Overall, 4.3\% ( $\mathrm{n}=5)$ patients were hyperthyroid and 71.8\% ( $n=84$ ) of patients were hypothyroid at 6-month followup. On long-term follow-up, $16.2 \%(n=19)$ of patients were found to have relapse of hyperthyroidism. All patients who relapsed underwent ST. Compared with TT, patients who underwent ST were 1.63 [odds ratio (OR) $1.63,95 \%$ confidence interval (CI) 1.40-1.91] times as likely to experience disease relapse. Fifty out of 79 patients in the ST group (63\%) required thyroxine replacement as compared with $100 \%$ of patients in the TT group.

\section{Postoperative Hypocalcemia}

Among the patients, 55.6\% $(n=65)$ developed hypocalcemia postthyroid surgery. Among these patients, hypocalcemia was temporary in 48 patients $(41.0 \%)$ and resolved within 6 months. The rest of the 17 patients had permanent hypocalcemia and required regular calcium and vitamin D supplementation. Undergoing TT was associated with higher odds of developing hypocalcemia postoperatively overall (OR 1.96; 95\% CI 1.08-3.57), but did not independently predict for both temporary $(p=0.229)$ and permanent hypocalcemia $(p=0.174)$.

\section{Postoperative Hoarseness and Recurrent Laryngeal Nerve Injury}

No intraoperative identification of recurrent laryngeal nerve injury was identified during this case series. The incidence of postoperative hoarseness was negligible in both ST and TT groups; $2.5 \%(n=2)$ and 3.3\% $(n=1)$ of patients post-TT experienced hoarseness; 2 out of the 3 cases were performed without neuromonitoring. All three cases of postoperative hoarseness were transient and the patients recovered without active intervention.

\section{Length of Hospitalization}

Patients had slightly shorter hospitalization post-ST compared with patients who underwent TT $(3.58 \pm 1.12$ vs $3.84 \pm 3.404$ days), but this did not reach statistical significance $(p=0.541)$.

\section{Factors predicting Failure of Therapy in Patients undergoing ST}

Table 2 demonstrates the preoperative values of thyroid function tests and antithyroid antibodies performed prior to patients undergoing ST. There was no significant difference between the preoperative levels of TSH, thyroxine, TSH receptor antibody, thyroid peroxidase, and antithyroglobulin between patients who experienced treatment 
Table 2: Comparison of pre-operative and operative factors affecting disease relapse post subtotal thyroidectomy

\begin{tabular}{llll}
\hline Parameters & $\begin{array}{l}\text { No disease } \\
\text { relapse }\end{array}$ & $\begin{array}{l}\text { Disease } \\
\text { relapse }\end{array}$ & $p$-value \\
\hline TSH (mIU/L) & $0.934 \pm 3.23$ & $0.093 \pm 0.2$ & 0.289 \\
Thyroxine (pmol/L) & $58.9 \pm 114.6$ & $32.4 \pm 57.5$ & 0.349 \\
TRAB (IU/L) & $71.4 \pm 112.6$ & $23.5 \pm 23.4$ & 0.564 \\
Anti-TPO (IU/L) & $378 \pm 423$ & $362 \pm 493$ & 0.938 \\
Anti-TG (IU/L) & $455 \pm 958$ & $45.2 \pm 62.1$ & 0.352 \\
Size of gland (gm) & $118.7 \pm 84.3$ & $199.0 \pm 151$ & 0.024 \\
Remnant gland (gm) & $6.56 \pm 3.37$ & $6.27 \pm 1.74$ & 0.700 \\
\hline
\end{tabular}

failure compared with those who did not. Significantly, the mean thyroid volumes estimated by ultrasonography preoperatively were higher in patients who experienced relapse of hyperthyroidism $(\mathrm{p}=0.024)$.

\section{DISCUSSION}

\section{Key Results}

In our surgical series, patients with GD who underwent ST were almost twice as likely to experience recurrent hyperthyroidism compared with those who underwent TT. Significantly, patients who received TT had a higher risk of hypoparathyroidism although this did not translate into longer hospitalization stay that was statistically significant. We also demonstrated that patients with a large goiter were more likely to relapse after ST; preoperative thyroid function and thyroid antibody levels did not seem to exert any effect on treatment failure.

In our case series, we observed a change in the trend from ST to TT for GD between the years 2007 and 2009. This may have been secondary to the emerging literature in the early 2000s regarding the superiority of TT with regard to lesser risk of relapse of hyperthyroidism after TT. We observed that patients who underwent ST had significantly larger thyroid volumes compared with those who underwent TT. Although this trend may partially explain why patients who underwent ST had higher recurrent hyperthyroidism rates in our series, we feel that this may have been due to a greater acceptance by patients and lower threshold for referral for surgery in GD in the more recent years.

Three patients in the ST subgroup had persistent hyperthyroidism post-ST. In Lepner et $\mathrm{al}^{12}$ study, none of the patients after ST had recurrence of hyperthyroidism when followed up for a mean of 52 months. Annerbo et a $1^{18}$ described reduction of GD relapse from 20 to $3.3 \%$ after the surgical method was changed from ST to TT. The authors cite that in spite of TT, recurrence of hyperthyroidism occurred due to remnant tissue high up at the hyoid bone. Given the risk of remnant thyroid left behind at the hyoid bone area coupled with 2 to $4 \mathrm{gm}$ of thyroid left behind on both sides, the excessive amount of remnant thyroid gland may have contributed to the immediate treatment failure observed in the above three patients. In the subgroup of patients who underwent ST, we found that preoperative thyroid gland volume was a significant predictor for treatment failure even though remnant gland volume, preoperative thyroid status, and antibody levels were not. We observed that the amount of remnant gland between the groups of patients who had treatment failure did not differ significantly from those with no relapse. This supports our hypothesis that remnant thyroid tissue may have been left behind in areas of the neck that were not routinely explored, e.g., in the area of the hyoid bone.

Similar to the reported literature, patients who underwent TT in our series had significantly more hypocalcemia with no difference in rates of recurrent laryngeal nerve injury. Despite this, both patient groups had comparable length of stay postsurgery. This seems to suggest that even though patients were more likely to experience hypocalcemia post-TT, the severity of hypocalcemia was not significantly more severe than those who underwent $\mathrm{ST}$, as this would have necessitated intravenous calcium replacement which would have translated into longer hospitalization stay postoperatively.

Interestingly, we observed that over long-term followup, a large proportion (60\%) of our patients who received ST became hypothyroid and required thyroxine replacement. This would undermine the purpose of performing $\mathrm{ST}$, as one of the reported benefits of the procedure is the hope to maintain a euthyroid state without TSH suppression therapy or thyroxine replacement long term. A previous study demonstrated that hypothyroidism after ST occurred in a bimodal pattern ${ }^{19}$ and the mechanism of action is unclear ${ }^{19}$ even though it may be associated with longer preoperative antithyroid drug treatment and suppression of TSH. ${ }^{20}$ Possible theories of hypothyroidism after bilateral ST include insufficient remnant thyroid left behind after surgery. In cases where patients experienced late onset hypothyroidism, this is likely to be the natural disease progression of GD where the remnant thyroid gland eventually "burns out", rendering the patient hypothyroid.

\section{LIMITATIONS}

The main limitation of our study lies in it being a retrospective review, even though data were collected prospectively. Given that the patient population spanned over a period of 25 years, there were missing data (especially laboratory investigations) that dated to the preelectronic era and such data could not be easily retrieved. Furthermore, laboratory reference ranges may have 
changed over a period of 25 years and our study was not able to adjust for these changes.

We observed that patients receiving TT had a greater incidence of hypocalcemia, but TT predicted neither temporary nor permanent hypocalcemia. We believe that this may have been because our study was inadequately powered to show such a difference and a larger sample size would have demonstrated the effect of TT on both temporary and permanent hypoparathyroidism.

\section{CONCLUSION}

Our study findings are in agreement with what is already reported in the current literature, and current existing guidelines are also likely to be applicable in the Singaporean or Southeast Asian population. With our findings demonstrating lesser relapse of hyperthyroidism and similar safety profiles of TT in tandem with a high proportion of patients eventually needing thyroxine replacement after ST, we believe that TT should be the surgical procedure of choice for patients with GD. We find little reason to continue to offer ST as an alternative.

\section{REFERENCES}

1. Kurihara H. Total thyroidectomy for the treatment of hyperthyroidism in patients with ophthalmopathy. Thyroid 2002 Mar;12(3):265-267.

2. Lowery AJ, Kerin MJ. Graves' ophthalmopathy: the case for thyroid surgery. Surgeon 2009 Oct;7(5):290-296.

3. Chi SY, Hsei KC, Sheen-Chen SM, Chou FF. A prospective randomized comparison of bilateral subtotal thyroidectomy versus unilateral total and contralateral subtotal thyroidectomy for Graves' disease. World J Surg 2005 Feb;29(2):160-163.

4. Guo Z, Yu P, Liu Z, Si Y, Jin M. Total thyroidectomy vs bilateral subtotal thyroidectomy in patients with Graves' diseases: a meta-analysis of randomized clinical trials. Clin Endocrinol (Oxf) 2013 Nov;79(5):739-746.

5. Hoffmann R. Thyroidectomy in Graves' disease: subtotal, near total or total? Orbit 2009;28(4):241-244.

6. Hermann M, Roka R, Richter B, Freissmuth M. Early relapse after operation for Graves' disease: postoperative hormone kinetics and outcome after subtotal, near-total, and total thyroidectomy. Surgery 1998 Nov;124(5):894-900.

7. Catania A, Guaitoli E, Carbotta G, Bianchini M, Di Matteo FM, Carbotta S, Nardi M, Fabiani E, Grani G, D'Andrea V, et al. Total thyroidectomy for Graves' disease treatment. Clin Ter 2013 May-Jun;164(3):193-196.

8. Barakate MS, Agarwal G, Reeve TS, Barraclough B, Robinson B, Delbridge LW. Total thyroidectomy is now the preferred option for the surgical management of Graves' disease. ANZ J Surg 2002 May;72(5):321-324.

9. Bahn Chair RS, Burch HB, Cooper DS, Garber JR, Greenlee MC, Klein I, Laurberg P, McDougall IR, Montori VM, Rivkees SA, et al. Hyperthyroidism and other causes of thyrotoxicosis: management guidelines of the American Thyroid Association and American Association of Clinical Endocrinologists. Thyroid 2011 Jun;21(6):593-646.

10. RobertJ,MariéthozS, PacheJC, Bertin D, Caulfield A, MurithN, Peytremann A, Goumaz M, Garcia B, Martin-Du Pan R, et al. Short- and long-term results of total vs subtotal thyroidectomies in the surgical treatment of Graves' disease. Swiss Surg 2001 Feb;7(1):20-24.

11. Werga-Kjellman P, Zedenius J, Tallstedt L, Träisk F, Lundell G, Wallin G. Surgical treatment of hyperthyroidism: a ten-year experience. Thyroid $2001 \mathrm{Feb} ; 11(2): 187-192$.

12. Lepner U, Seire I, Palmiste V, Kirsimägi U. Surgical treatment of Graves' disease: subtotal thyroidectomy might still be the preferred option. Medicina (Kaunas) 2008;44(1):22-26.

13. Witte J, Goretzki PE, Dotzenrath C, Simon D, Felis P, Neubauer M, Röher HD. Surgery for Graves' disease: total versus subtotal thyroidectomy-results of a prospective randomized trial. World J Surg 2000 Nov;24(11):1303-1311.

14. Jarhult J, Rudberg C, Larsson E, Selvander H,Sjövall K, Winsa B, Rastad J, Karlsson FA; TEO Study Group. Graves' disease with moderate-severe endocrine ophthalmopathy-long term results of a prospective, randomized study of total or subtotal thyroid resection. Thyroid 2005 Oct;15(10):1157-1164.

15. Liu ZW, Masterson L, Fish B, Jani P, Chatterjee K. Thyroid surgery for Graves' disease and Graves' ophthalmopathy. Cochrane Database Syst Rev 2015 Nov;11:CD010576.

16. KumaK, Matsuzuka F, Kobayashi A,HiraiK, FukataS, TamaiH, Miyauci A, Sugawara M. Natural course of Graves' disease after subtotal thyroidectomy and management of patients with postoperative thyroid dysfunction. Am J Med Sci 1991 Jul;302(1):8-12.

17. Sugino K, Mimura T, Ozaki O, Kure $\mathrm{Y}$, Iwasaki H, Wada N, Matsumoto A, Ito K. Early recurrence of hyperthyroidism in patients with Graves' disease treated by subtotal thyroidectomy. World J Surg 1995 Jul-Aug;19(4):648-652.

18. Annerbo M, Stalberg P, Hellman P. Management of Grave's disease is improved by total thyroidectomy. World J Surg 2012 Aug;36(8):1943-1946.

19. Hedley AJ, Bewsher PD, Jones SJ, Khir AS, Clements P, Matheson NA, Gunn A. Late onset hypothyroidism after subtotal thyroidectomy for hyperthyroidism: implications for long term follow-up. Br J Surg 1983 Dec;70(12):740-743.

20. Nonchev BI, Terzieva DD, Dimov RS, Mateva NG, Tsvetkova TZ, Dimitrakov DY. Early hypothyroidism after subtotal thyroidectomy in patients with Graves' disease-the role of the preoperative conservative treatment and hormonal status. Folia Med (Plovdiv) 2005;47(3-4):40-45. 